\title{
Epidemiology of Dengue Fever: A Model with Temporary Cross-Immunity and Possible Secondary Infection Shows Bifurcations and Chaotic Behaviour in Wide Parameter Regions
}

\author{
Maíra Aguiar ${ }^{a, c, d}$, Bob Kooi ${ }^{b}$ and Nico Stollenwerk ${ }^{a, c} 1$ \\ ${ }^{a}$ Centro de Matemática e Aplicações Fundamentais da Universidade de Lisboa, \\ Avenida Prof. Gama Pinto 2, 1649-003 Lisboa, Portugal, \\ ${ }^{b}$ Department of Theoretical Biology, Vrije Universiteit, \\ De Boelelaan 1087, NL 1081 HV Amsterdam, The Netherlands, \\ ${ }^{c}$ Instituto Gulbenkian de Ciência, Apartado 14, 2781-901 Oeiras, Portugal, \\ ${ }^{d}$ Laboratório de dengue e febre amarela, Fundação Ezequiel Dias, \\ Rua Conde Pereira Carneiro 80, 30510-010 Belo Horizonte-MG, Brasil
}

\begin{abstract}
Basic models suitable to explain the epidemiology of dengue fever have previously shown the possibility of deterministically chaotic attractors, which might explain the observed fluctuations found in empiric outbreak data. However, the region of bifurcations and chaos require strong enhanced infectivity on secondary infection, motivated by experimental findings of antibody-dependent-enhancement. Including temporary cross-immunity in such models, which is common knowledge among field researchers in dengue, we find bifurcations up to chaotic attractors in much wider and also unexpected parameter regions of reduced infectivity on secondary infection, realistically describing more likely hospitalization on secondary infection when the viral load becomes high. The model shows Hopf bifurcations, symmetry breaking bifurcations of limit cycles, coexisting isolas, and two different possible routes to chaos, via the Feigenbaum period doubling and via torus bifurcations.
\end{abstract}

Key words: temporary cross-immunity, antibody-dependent-enhancement (ADE), Lyapunov exponents, torus bifurcation, isolas.

AMS subject classification: 37G35, 37D45, 65P30, 92B05

${ }^{1}$ Corresponding author. E-mail: nico@ptmat.fc.ul.pt 


\section{Introduction}

Dengue fever is caused by four antigenically distinct viruses, designated dengue types $1,2,3$, and 4 [35]. Infection by one serotype confers life-long immunity to only that serotype and estimated three to nine months of temporary cross-immunity to other serotypes $[22,33,7,24]$. The empiric time of temporary cross-immunity is mainly based on detectable antibody levels. However, the epidemiological period of temporary cross-immunity can be much larger [22, 34].

Among symptomatic cases dengue fever (DF) is often benign. But a severe form known as dengue hemorrhagic fever (DHF), which may evolve towards dengue shock syndrome (DSS) can also occur. Without proper treatment DHF/DSS case fatality rates can exceed $20 \%$ [35]. There are indeed pre-existing antibodies to previous dengue virus that cannot neutralize but rather enhance infection in vitro, a process described as antibody-dependent enhancement (ADE). The ADE theory states that cross-reactive, non-neutralizing antibodies from a previous heterologous dengue virus infection bind to the new infecting serotype and facilitate virus entry via Fc-receptor-bearing cells such as monocytes and macrophages. Increased virus replication and antigen presentation lead to an exaggerated immune response increasing disease manifestation with plasma leakage and haemorrhagic phenomenae [20, 17, 9, 23]. Epidemiological studies support the association of DHF with secondary dengue infection $[16,8,35,18,19]$. However, there is no animal model of DHF/DSS.

Mathematical models describing the transmission of dengue viruses appeared in the literature as early as 1970 [14]. More recently, modelling attention has focussed on higher viral load of hosts on secondary infection than on the first due to ADE, hence a higher contribution to the force of infection of each strain, reporting deterministically chaotic attractors [13] and chaos desynchronization $[30,6]$ to explain the co-existence of the known four dengue viral strains. Temporary cross-immunity against all strains after a first infection has been included in mathematical models as well, but again limiting the effect of ADE to increase the contribution of secondary cases to the force of infection [33]. To our knowledge, no systematic investigation of the attractor structures of simple multi-strain models with dengue-realistic temporary cross-immunity and decreased contribution of secondary infection to the force of infection, due to severity of infection with a second strain caused by higher viral load and eventual hospitalization, has been performed so far. Temporary cross-immunity also has to be distinguished from partial cross-immunity as also modelled for dengue $[1,2]$.

We investigate a basic two-strain model, initially suggested and preliminarily analysed in [3], to capture primary and secondary infection, with main attention to differences in the force of infection in primary versus secondary infection (parametrized by $\phi$ ) and the effect of temporary cross-immunity between the first and second infection with distinct strains (parametrized by $\alpha$ ). Neglecting the effect of temporary cross-immunity or considering a very short period of one week (transition rate $\alpha=52 /$ year) we find the first Hopf bifurcation from a steady state to a limit cycle, hence non-equilibrium dynamic behaviour, for a more that one and a half times higher infectivity on secondary infection versus primary (ratio $\phi>1.5$ ). Whereas including a realistic value for the temporary cross-immunity of e.g. half a year $(\alpha=2 /$ year $)$, we find the first Hopf bifurcation for the infectivity ratio as low a one tenth $(\phi=0.1)$ and a positive Lyapunov exponent as sign of a 
deterministically chaotic attractor around $\phi=0.5$.

An extremely rich bifurcation structure is observed for $\phi<1$ when taking the temporary cross-immunity in a dengue realistic parameter regime for $\alpha$ between one to three. Improving earlier presented results [3] here we explore and describe in more detail the rich bifurcation structure around such low $\alpha$ values, especially $\alpha=2$ (in the following we omit the unit per year for the rates) and $\phi<1$. In this parameter region the model shows Hopf bifurcations, symmetry breaking bifurcations of limit cycles, coexisting isolas, and two different possible routes to chaos, via the Feigenbaum period doubling and via torus bifurcations. Whereas previous modelling efforts have concentrated on $\phi>1$ we find this rich dynamics when in the secondary infection people are less infectious than people in first dengue infection, hence for $\phi<1$. This assumption is likely to be more realistic for dengue fever since the possible severity of a secondary infection may hospitalize people, not contributing to the force of infections as much as people with first infection. Nevertheless, the relatively restrictive assumption of much higher contribution to the force of infection of secondary infectivity previously necessary for complex dynamics can be relaxed significantly when taking the temporary cross-immunity into account.

Hence observed fluctuations in dengue outbreak data could now be understood better considering multi-strain dynamics as significant factor. The more detailed understanding of possible state space scenarios through bifurcation analysis will help in future understanding of dengue epidemiological data and its multi-strain aspects. The basic model structure allows to generalize our findings to other multi-strain epidemiological systems expecting the same complexity.

\section{Basic two-strain epidemic model}

The present model is a basic two-strain SIR-type model dividing the host population into susceptible (S), infected (I) and recovered individuals (R). It can be understood as a mean field approximation of a stochastic system. The simple SIR epidemics without strain structure of the pathogens reads

$$
\begin{aligned}
\dot{S} & =\alpha R-\frac{\beta}{N} \cdot I \cdot S+\mu(N-S) \\
\dot{I} & =\frac{\beta}{N} \cdot I \cdot S-\gamma I-\mu I \\
\dot{R} & =\gamma I-\alpha R-\mu R
\end{aligned}
$$

for a host population of $N$ individuals, with contact and infection rate $\beta$, recovery rate $\gamma$ and temporary immunity rate $\alpha$. Demography is denoted as exits from all classes $S, I$ and $R$ with rate $\mu$ to the new born susceptibles. The system has only equilibria steady solutions as attractors. Transients under certain parameter values oscillate into the equilibrium, hence can be already more complex than the final attractor. Stochastic versions of such models with only fixed points possible 
as attractors but oscillating transients are reported to also show stabilization of the oscillations due to population noise $[5,25]$.

To capture differences in primary infection by one strain and secondary infection by another strain we consider a basic two-strain SIR-type model for the host population, which is only slightly refined as opposed to previously suggested models for dengue fever [13, 30]. It is capturing the effective dynamics of the human host population for the dengue virus, taking effects of the vector dynamics or seasonality only into account by the effective parameters in the SIR-type model, but not modelling these mechanisms explicitly. Instead we focus on the multi-strain aspect and its effects on the host population. The complete system of ordinary differential equations for the two strain epidemiological system is given by

$$
\begin{aligned}
\dot{S} & =-\frac{\beta_{1}}{N} S\left(I_{1}+\phi_{1} I_{21}\right)-\frac{\beta_{2}}{N} S\left(I_{2}+\phi_{2} I_{12}\right)+\mu(N-S) \\
\dot{I}_{1} & =\frac{\beta_{1}}{N} S\left(I_{1}+\phi_{1} I_{21}\right)-(\gamma+\mu) I_{1} \\
\dot{I}_{2} & =\frac{\beta_{2}}{N} S\left(I_{2}+\phi_{2} I_{12}\right)-(\gamma+\mu) I_{2} \\
\dot{R_{1}} & =\gamma I_{1}-(\alpha+\mu) R_{1} \\
\dot{R_{2}} & =\gamma I_{2}-(\alpha+\mu) R_{2} \\
\dot{S_{1}} & =-\frac{\beta_{2}}{N} S_{1}\left(I_{2}+\phi_{2} I_{12}\right)+\alpha R_{1}-\mu S_{1} \\
\dot{S_{2}} & =-\frac{\beta_{1}}{N} S_{2}\left(I_{1}+\phi_{1} I_{21}\right)+\alpha R_{2}-\mu S_{2} \\
\dot{I_{12}} & =\frac{\beta_{2}}{N} S_{1}\left(I_{2}+\phi_{2} I_{12}\right)-(\gamma+\mu) I_{12} \\
\dot{I_{21}} & =\frac{\beta_{1}}{N} S_{2}\left(I_{1}+\phi_{1} I_{21}\right)-(\gamma+\mu) I_{21} \\
\dot{R} & =\gamma\left(I_{12}+I_{21}\right)-\mu R .
\end{aligned}
$$

For two different strains, 1 and 2, we label the SIR classes for the hosts that have seen the individual strains. Susceptibles to both strains $(\mathrm{S})$ get infected with strain $1\left(I_{1}\right)$ or strain $2\left(I_{2}\right)$, with force of infection $\beta_{1}$ and $\beta_{2}$ respectively. They recover from infection with strain 1 (becoming $R_{1}$ ) or from strain 2 (becoming $R_{2}$ ), with recovery rate $\gamma$. In this recovered class, people have full and life-long immunity against the strain that they were exposed to and infected, and also a period of temporary cross-immunity against the other strain. After this, with rate $\alpha$, they enter again in the susceptible classes ( $S_{1}$ respectively $S_{2}$ ), where the index represents the first infection strain. Now, 
$S_{1}$ can be reinfected with strain 2 (becoming $I_{12}$ ), meeting $I_{2}$ with infection rate $\beta_{2}$ or meeting $I_{12}$ with infection rate $\phi_{2} \beta_{2}$, secondary infected contributing differently to the force of infection than primary infected, and $S_{2}$ can be reinfected with strain 1 (becoming $I_{21}$ ) meeting $I_{1}$ or $I_{21}$ with infections rates $\beta_{1}$ and $\phi_{1} \beta_{1}$ respectively.

The parameter $\phi$ in our model, as opposed to the previous dengue models, acts decreasing the infectivity of secondary infection, once people with higher viral load are more likely to be hospitalized because of the severity of the disease (DHF/DSS), and do not contributed to the force of infection as much as people with first infection do. Finally, $I_{12}$ and $I_{21}$ go to the recovered class (R), immune against all strains. We include demography of the host population denoting the birth and death rate by $\mu$. For constant population size $N$ we have $R=N-\left(S+I_{1}+I_{2}+R_{1}+R_{2}+\right.$ $\left.S_{1}+S_{2}+I_{12}+I_{21}\right)$ and therefore we only need to consider the first 9 equations of Eq. system (2.2). In our numerical studies we take the population size equal to $N=100$ so that numbers of susceptibles, infected etc., are given in percentage.

To take biological information from experiences in dengue into account we fix the transition rates of the model as far as is known, and only will vary the most unknown parameter $\phi$. For simplicity, we consider $\phi_{1}=\phi_{2}=\phi, \beta_{1}=\beta_{2}=\beta$, i.e, no epidemiological asymmetry between strains. The parameter values are given in Table 1 , if not otherwise explicitly stated.

Table 1: Parameter set, rates given in units per year, ratio without unit

\begin{tabular}{lll}
\hline Par. & Description & Values \\
\hline$N$ & population size & 100 \\
$\mu$ & new born susceptible rate & $1 / 65$ \\
$\gamma$ & recovery rate & 52 \\
$\beta_{1}=\beta_{2}=\beta$ & infection rate & $2 \gamma$ \\
$\alpha$ & temporary cross-immuntiy rate & 2 \\
$\phi_{1}=\phi_{2}=\phi$ & ratio of contrib. to force of inf. & variable
\end{tabular}

\section{Time series analysis}

In this section we investigate time series simulations of the present model, Eq. system (2.2). We performed a detailed analysis of the attractor structure, investigating state space plots for various values of $\phi$. Besides the previously analyzed region of $\phi>1$ we also observe a rich dynamical behaviour from fixed points to bifurcating limit cycles and chaotic attractors for $\phi<1$. Maxima return maps are evaluated from extremely long time serie, and Lyapunov exponents are calculated. This rich dynamic structure will be analyzed in the next section in more detail via bifurcation analysis by continuation. 


\subsection{Time series simulations}

In order to classify the dynamic pattern of the model for various parameters, we discard long transients which would carry information about the initial conditions. In the following simulations we discarded the first 2000 years (see Fig. 1). However, also the transients reflect the dynamic behaviour of the system under the present parameter values.

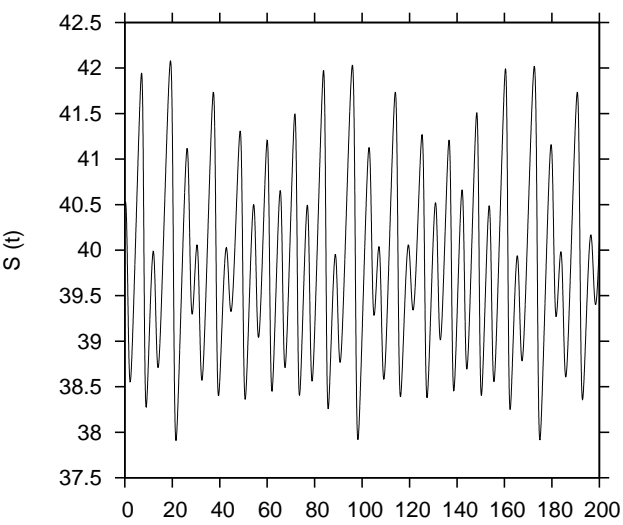

a)

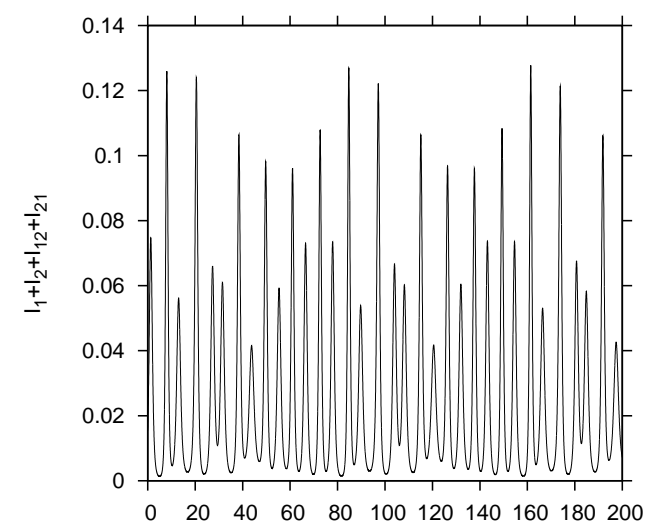

b)

Figure 1: For $\alpha=2$ and $\phi=0.6$, time series simulations of a) the suceptibles $S$, and in b) the total number of infected $I$.

The time series for $\phi<1$, as would be realistic for dengue fever due to more severe disease upon reinfection and larger chance of people being hospitalized, shows that the total number of infected

$$
I:=I_{1}+I_{2}+I_{12}+I_{21}
$$

stays quite away from zero, avoiding the chance of extintion in stochastic systems with reasonable system size (see Fig. 1 b)).

The parameter region previously considered to model ADE effects on dengue epidemiology, i.e. $\phi>1$, leads to rather low troughs for the total number of infected giving unrealistically low numbers of infected. In Fig. 2 a) the logarithm of total number of infected goes as low as -70 for $\phi=2.7$ in the chaotic region of $\phi>1$. Population fluctuations would in this case drive almost surely the system to extinction.

For $\phi=0.6$, hence the chaotic dynamics in the region of $\phi<1$, see Fig. 2 b), the logarithm of total infected does not pass below -7 . This encourages us to look closer to the parameter region of $\phi<1$, when dengue patients with severe disease because of the ADE phenomenon contribute less to the force of infection due to possible hospitalization, and not more, as previous models suggested.

\subsection{State space plots}

Next, we investigate the state space plots in terms of the variables $S$ and the logarithm of the total number of infected $I$, since dengue notification data often do not distinguish between the 


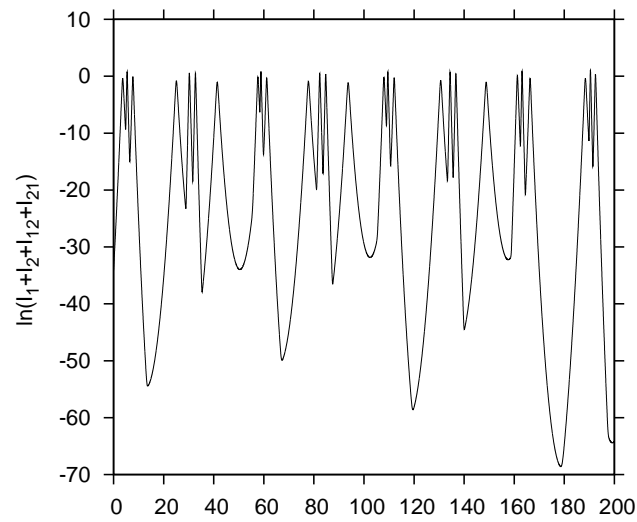

a)

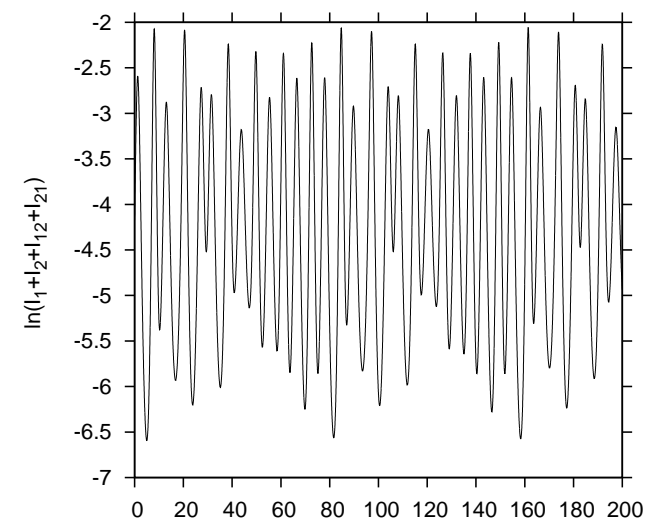

b)

Figure 2: Time series of the logarithm of the overall infected $(\ln (I))$ comparisson: a) simulation for $\phi=2.7$ and $\mathrm{b}$ ) simulation for $\phi=0.6$ for the same time interval.

circulating strains, whereas the susceptible class $S$ is $N$ minus every host who ever has experienced an infection, an information which eventually can be obtained from serological studies. In eventual data analysis the method of delay coordinates even allows to only work with one time series of $I$, and analysing $I(t), I(t+\tau)$ etc., with a time delay $\tau$ obtaining full topological information of the attractor structure [27, 12].

Varying $\phi$, the state space plots show a rich dynamical behaviour with bifurcations from fixed point to limit cycles, until completely irreguar behaviour, which is the fingerprint of deterministc chaos (see Fig. 3).

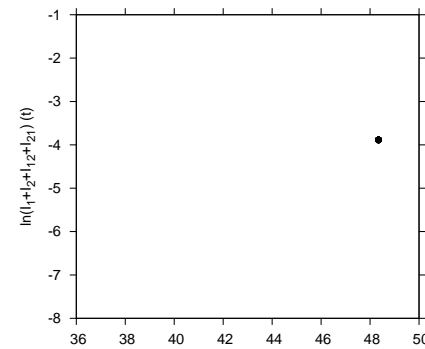

a) b)

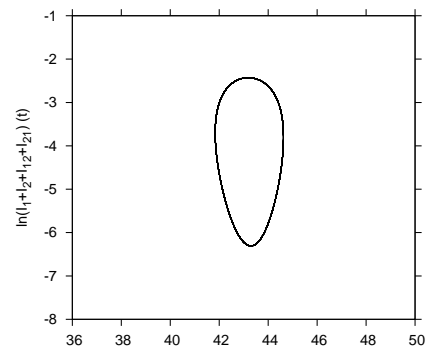

$\mathrm{S}(\mathrm{t})$

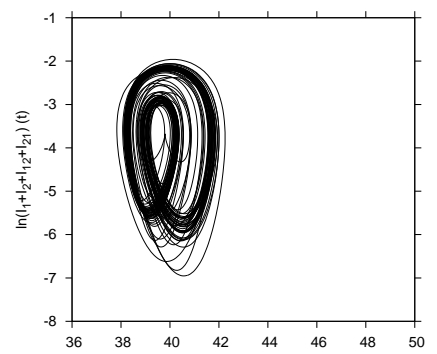

c) $\mathrm{S}(\mathrm{t})$

Figure 3: Attractors for various values of $\phi<1$ : a) fixed point for $\phi=0.1$, and b) limit cycle for $\phi=0.4$, and c) chaotic attractor for $\phi=0.6$.

Looking for higher values of $\phi$, the chaotic attractor becomes unstable, just leaving simple limit cycles as attractors for large parameter regions beyond $\phi=1$ [3]. Only for much higher values of $\phi>>1$, another chaotic attractor appears, the classical "ADE chaotic attractor" [13, 30, 3]. 


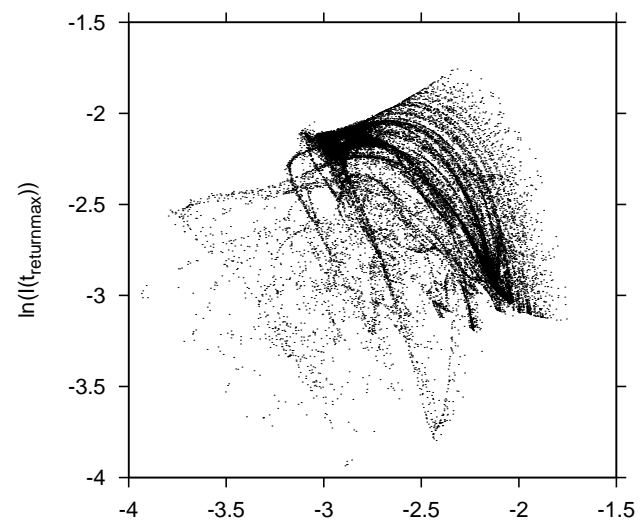

a)

$$
\ln \left(I\left(t_{\max }\right)\right)
$$

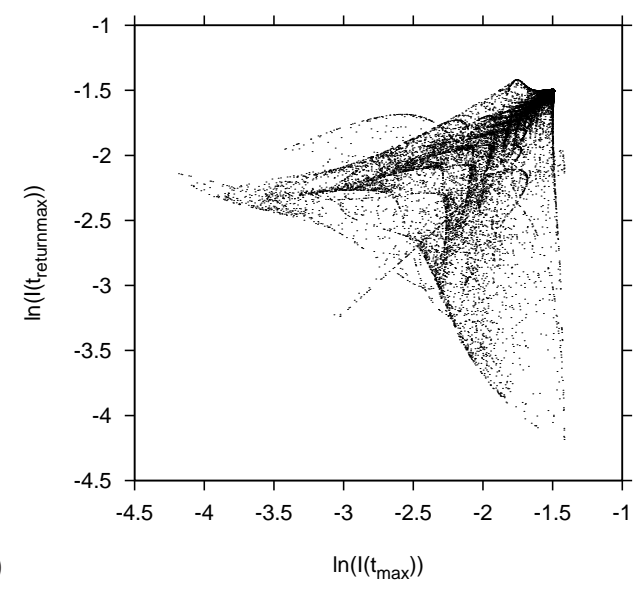

b)

Figure 4: Maxima return map for $\alpha=2$ and 200000 years of transients discarded. Deterministically chaotic attractors for a) $\phi=0.6$ and b) $\phi=0.99$ are observed.

\subsection{Maxima return map of $I$ from state space plot}

We investigate maxima return maps in order to classify the dynamics for various parameter values from extremely long time series. For the time $t_{\max }$, at which the total number of infected $I(t)$ has a local maximum, we plot the logarithm of the number of infected at that time $\ln \left(I\left(t_{\max }\right)\right)$ and at the next local maximum $\ln \left(I\left(t_{\text {returnmax }}\right)\right)$ (see Fig. 4).

We discarded long transients and ploted 200000 years of simulation. A deterministically chaotic attractor was obtained from our two-strain model with temporary cross-immunity in the region of $\phi<1$, where the secondary infection contributes less than the first infection to the overall force of infection. We observed that even after 400000 years, the dots never come back to the same point, so the fingerprint of chaotic attractors is clearly visible now.

\subsection{Numerical bifurcation diagram}

The bifurcation diagram was obtained ploting the local extrema of $\ln (I)$ over the varying parameter $\phi$ (see Fig. 5). Fixed points appear as one dot per parameter value, limit cycles appear as two dots, double-limit cycles as four dots, more complicated limit cyles as more dots, and chaotic attractors as continuously distributed dots for a single $\phi$ value [29].

We observe a chaotic window for $\phi<1$ where this dynamical behaviour has never been described before, and also another one for $\phi>1$, where the minimal values go to very low numbers of infected, the classical "ADE chaotic region", which already has been described in previous publications $[13,30,6]$.

However, to be sure that this unexpeted behaviour for $\phi<1$ not just appears because of this specific $\alpha$ value, we look at the robustness of the findings by varying the temporary cross-immunity parameter values. For $\alpha=1 \mathrm{e} . \mathrm{g}$ (which would be also acceptable for dengue when we realize that because of seasonality of the disease, people generally do not get sick more than once per year), both chaotic windows appear, and surprisingly in the region of $\phi<1$ this window is even larger 


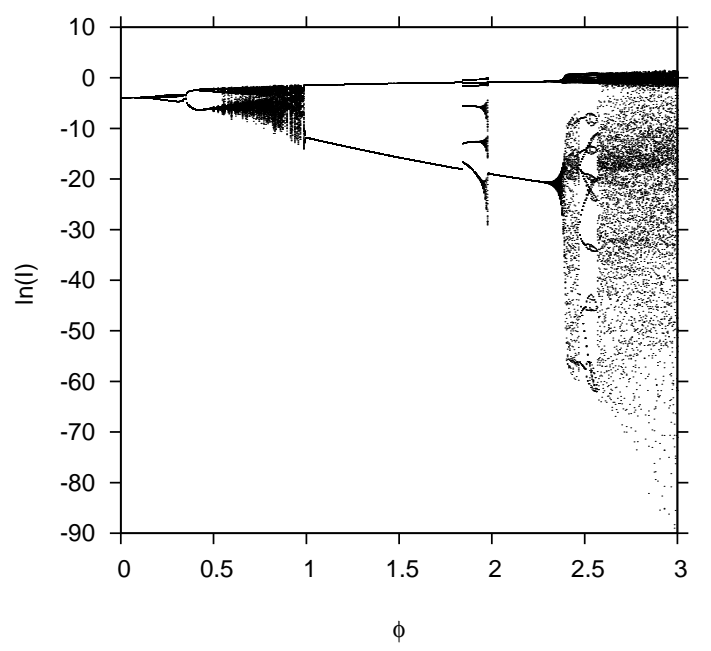

Figure 5: Bifurcation diagram for the local extrema of the overall infected with changing parameter $\phi$ and fixed $\alpha=2$. Here, 2000 years of transients were discarded.

(see Fig. 6 a)). The bifurcation diagram appears to be quite robust against changes of parameters around the region under investigation, in the sense that it shows chaotic windows for $\phi<1$ and for $\phi>1$.

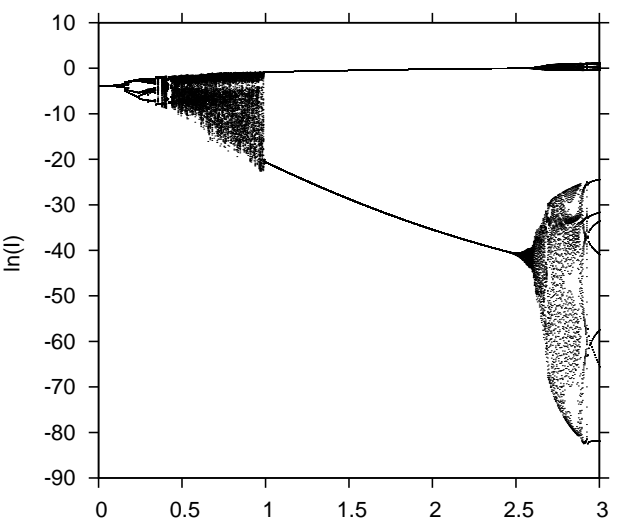

a)

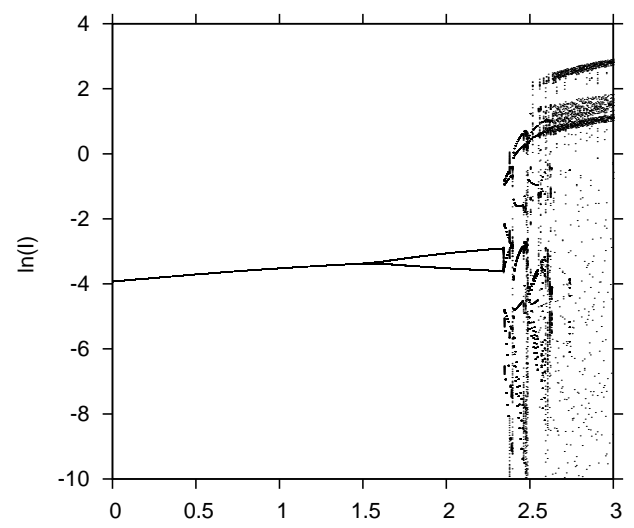

b)

Figure 6: Bifurcation diagram for the extrema of the overall infected with changing parameter $\phi$. In a) $\alpha=1$ and in b) $\alpha=52$ or inverse one week. Only the upper part of the bifurcation diagram is shown. The minima in $\ln (I)$ go down as low as -400 in logarithmic scale.

For very large values of $\alpha$, we get close to the models found in the literature, where temporary cross-immunity becomes shorter or unimportant due to the low resident times in the classes $R_{1}$ and $R_{2}$. The chaotic window for $\phi<1$ disappears, and then ADE as increasing infectivity on a secondary infection condition seems to be the only mechanism to observe deterministic chaos (see Fig. $6 \mathrm{~b}$ )). We observed again that for $\phi>1$ the number of infected goes to very low troughs, whereas in the chaotic region for $\phi<1$ and $\alpha=2$, the overall number of infected stays always 
sustainably high, i.e. never goes lower than -15 in logarithmic scale (see Fig. 5).

In Fig. 6 it becomes clear that for larger $\alpha$ (for vanishing temporary cross-immunity), there is no other dynamics in the region for $\phi<1$, than equilibria or limit cycles, the reason why chaos for $\phi<1$ has not been observed before.

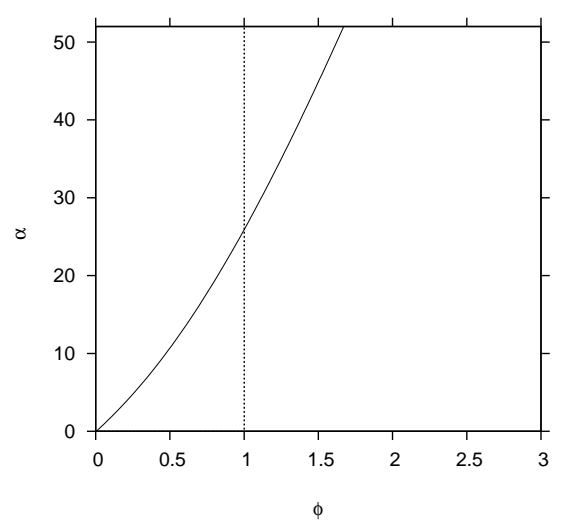

Figure 7: Line of the Hopf bifurcation from stable fixed point to limit cycle in the $\phi-\alpha$ plane. Here we clearly see that in the region of $\phi<1$ the Hopf bifurcation happens when the temporary cross immunity is considerable $(\alpha<20)$. When the temporary cross-immunity is less significant $(\alpha>30)$, the Hopf bifurcation point appears only in the region of $\phi>1$.

This observation is further confirmed by a two-parameter bifurcation diagram where $\phi$ and $\alpha$ are the free parameters, see Fig. 7. The Hopf bifurcation line in the $\phi-\alpha$ plane only shows Hopf bifurcation to limit cycles in the region of $\phi<1$ untill $\alpha \approx 20$, where the temporary crossimmunity period is still considerable. For $\alpha>20$, i.e. less significant temporary cross-immunity period, the Hopf bifurcation existis only in the $\phi>1$ region which was described in the literature before. There is a also sharp bend of the Hopf bifurcation line for very small $\alpha$ values close to the origin and the bifurcation curve continues close to the horizontal axis for increasing $\phi$. However this region is of no biological importance since here the parameter $\alpha$ is even smaller than the birth and death rate $\mu$.

\subsection{Quantifying unpredictability: Lyapunov exponents}

We now quantify the attractor structure, fixed point, limit cycle or chaotic attractor etc., by calculating Lyapunov exponents $[29,26]$. A negative largest Lyapunov exponent indicates a stable fixed point as attractor, a zero largest Lyapunov exponent indicates a stable limit cycle and a positive largest Lyapunov exponent indicates a chaotic attractor.

As short hand notation for Eq. system (2.2) let the dynamics for the state

$$
\underline{x}:=\left(S, I_{1}, I_{2}, \ldots, R\right)
$$

be $\underline{f}(\underline{x})$, hence

$$
\frac{d}{d t} \underline{x}=\underline{f}(\underline{x})
$$


which explicitly gives the dynamics as written down above. Then we analyse the stability in all 9 directions of the state space of this ODE system by calculating deviations $\Delta \underline{x}$ along a numerically integrated solution of Eq. (3.3) in the attractor with attractor trajectory $\underline{x}^{*}(t)$, hence

$$
\frac{d}{d t} \Delta \underline{x}=\left.\frac{d \underline{f}}{d \underline{x}}\right|_{\underline{x}^{*}(t)} \cdot \Delta \underline{x} .
$$

Here, any attractor is notified by $\underline{x}^{*}(t)$, be it a fixed point, periodic orbit or chaotic attractor. In this ODE system the linearized dynamics is given with the Jacobian matrix $\frac{d f}{d \underline{x}}$ of the ODE system Eq. (3.3) evaluated at the trajectory points $\underline{x}^{*}(t)$ given in notation of $\left.\frac{d f}{d \underline{x}}\right|_{\underline{x^{*}}(t)}$.

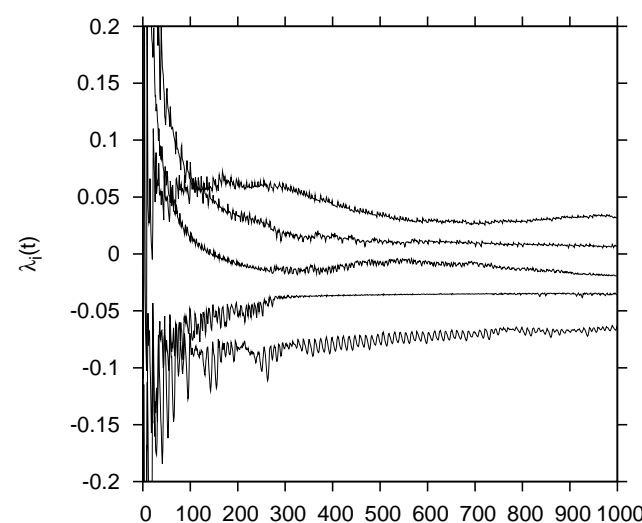

a)

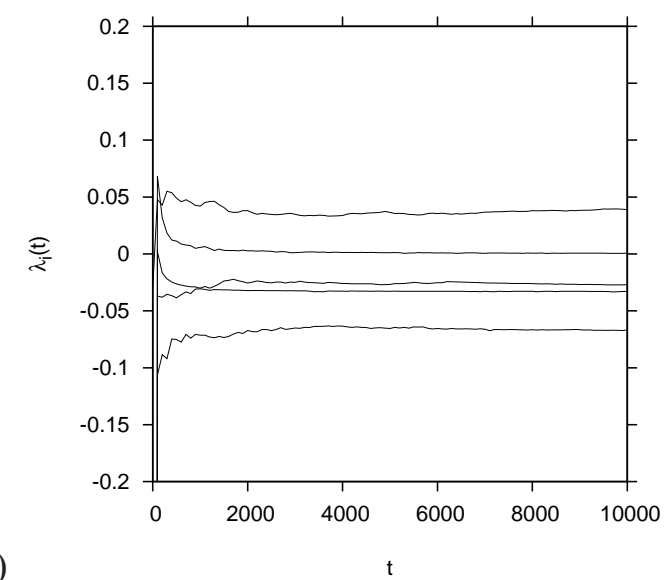

Figure 8: Lyapunov exponents measuring chaoticity of the attractor a) along short trajectory fast converging to qualitatively right behaviour, b) along longer trajectory for higher numerical precision. The five largest Lyapunov exponents are shown. Parameters are $\alpha=2$ and $\phi=0.6$.

The Lyapunov exponents then are the logarithms of the eigenvalues of the integrated Eq. (3.4) in the limit of large integration times. Besides for very simple iterated maps no analytic expressions for chaotic systems can be given for the Lyapunov exponents. For the calculation of the iterated Jaconbian matrix and its eigenvalues, we use the QR decomposition algorithm $[11,28]$. With the matrix $A\left(\underline{x}^{*}(t)\right):=\nVdash+\left.\Delta t \frac{d \underline{f}}{d \underline{x}}\right|_{\underline{x}^{*}(t)}=Q\left(\underline{x}^{*}(t)\right) \cdot R\left(\underline{x}^{*}(t)\right)$, where $\nVdash$ is the unit $(9 \times 9)$-matrix, we have

$$
\begin{aligned}
\Delta \underline{x}\left(t_{0}+(n+1) \Delta t\right) & =A_{n} \cdot A_{n-1} \cdot \ldots \cdot A_{0} \cdot \Delta \underline{x}\left(t_{0}\right) \\
& =Q_{n} \cdot R_{n} \cdot R_{n-1} \cdot \ldots \cdot R_{0} \cdot \Delta \underline{x}\left(t_{0}\right)
\end{aligned}
$$

for $A_{n}=A\left(\underline{x}\left(t_{0}+n \Delta t\right)\right)$. From $R_{n} \cdot R_{n-1} \cdot \ldots \cdot R_{0}=\prod_{\nu=0}^{n} R_{\nu}$ with the diagonal elements $r_{i i}(\nu)$ of the right diagonal matrix $R_{\nu}$ the Lyapunov exponents are given for large $t=n \Delta t$ by

$$
\lambda_{i}(t)=\frac{1}{n \cdot \Delta t} \ln \left(\prod_{\nu=0}^{n}\left|r_{i i}(\nu)\right|\right) \text {. }
$$


Plots with $\lambda_{i}$ as function of time $t=n \Delta t$ are given in Fig. 8. For small integration times, see Fig. 8 a) the Lyapunov exponents change a lot along the attractor, but soon settle towards their final size, still showing small oscillations. For long integration times, see Fig. 8 b) these oscillations also disappear, giving reliable values for the infinit time limit of the Lyapunov exponents $\lambda_{i}=$ $\lim _{t \rightarrow \infty} \lambda_{i}(t)$.

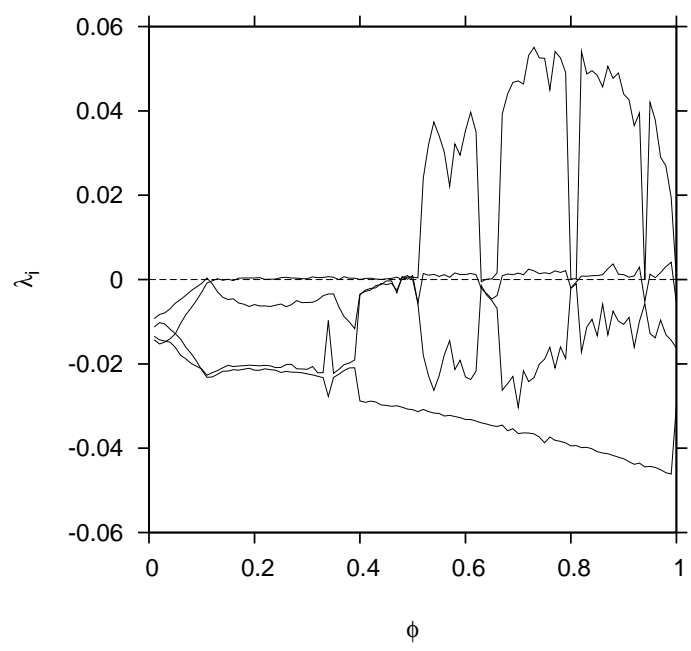

Figure 9: Spectrum of the four largest Lyapunov exponents with changing parameter $\phi$ and fixed $\alpha=2$.

Fig. 9 shows the largest four Lyapunov exponents as a function of $\phi$. We observe that for small $\phi$ up to 0.1 all four Lyapunov exponents are negative, indicating the stable fixed point solution. Then follows a region up to $\phi=0.5$ where the largest Lyapunov exponent is zero, characteristic for stable limit cycles. Above $\phi=0.5$ a positive Lyapunov exponent, clearly separated from the second largest Lyapunov exponent being zero, indicates deterministically chaotic attractors. In the chaotic window between $\phi=0.5$ and $\phi=1$ also periodic windows appear, giving a zero largest Lyapunov exponent. These findings are in good agreement with the numerical bifurcation diagram, and we will now further investigate this bifurcation structure in the next section.

\section{Bifurcation analysis by continuation}

In this section we give the analytic solution for the equilibria and describe the further analysis of the bifurcation structure, using numerical software like AUTO. In this case the bifurcation analysis is done by continuation techniques, i.e. starting from the equilibrium solution for small $\phi$ by following the solution for increasing $\phi$ and simultaneously the eigenvalue spectrum, until the eigenvalues show a loss of stability. At this point a Hopf bifurcation gives rise to a stable limit cycle, which subsequently is followed in parameter space, until it becomes unstable at the next bifurcation point etc. up to bifurcations which do not give limit cycles any more, like a torus bifurcation. Also accumulated period doubling bifurcations become increasingly difficult to follow. 
But first we can give an analytical solution for the equilibria which also serves as a cross check for the numerical programs.

\subsection{Stationary states for the symmetric case}

The stationary states can be calculated analytically by setting the time derivatives in Eq. system (2.2) to zero. For the symmetric case, i.e., $\beta_{1}=\beta_{2}=\beta$ and $\phi_{1}=\phi_{2}=\phi$ the stationary states are given by

$$
\begin{aligned}
S^{*} & =\frac{\mu N-(\gamma+\mu)\left(I_{1}^{*}+I_{2}^{*}\right)}{\mu} \\
I_{21}^{*} & =\frac{1}{\phi_{1}}\left(\frac{N}{\beta_{1} S^{*}}(\gamma+\mu+1)\right) I_{1}^{*} \\
I_{12}^{*} & =\frac{1}{\phi_{2}}\left(\frac{N}{\beta_{2} S^{*}}(\gamma+\mu+1)\right) I_{2}^{*} \\
S_{1}^{*} & =\frac{(\gamma+\mu) I_{12}^{*}}{\left(I_{2}^{*}+\phi_{2} I_{12}^{*}\right)} \frac{N}{\beta_{2}} \\
S_{2}^{*} & =\frac{(\gamma+\mu) I_{21}^{*}}{\left(I_{1}^{*}+\phi_{1} I_{21}^{*}\right)} \frac{N}{\beta_{1}} \\
R_{1}^{*} & =\frac{\gamma}{\alpha+\mu} I_{1}^{*} \\
R_{2}^{*} & =\frac{\gamma}{\alpha+\mu} I_{2}^{*},
\end{aligned}
$$

where still the stationary values of $I_{1}^{*}$ and $I_{2}^{*}$ have to be determined.

The solution of coexistence of both strains for $I_{1}=I_{2}=I^{*}$ is given by the following expression

$$
\begin{aligned}
I_{1,2}^{*} & =-\left[\frac{\frac{\alpha \gamma}{(\alpha+\mu)(\gamma+\mu)} \phi+\left(\frac{(\gamma+\mu)}{\beta}-3\right)}{4 \frac{(\gamma+\mu)}{\mu}\left(1-\frac{\alpha \gamma}{(\alpha+\mu)(\gamma+\mu)} \phi\right)}\right] N \\
& -\sqrt{\frac{N^{2}}{4}\left[\frac{\frac{\alpha \gamma}{(\alpha+\mu)(\gamma+\mu)} \phi+\left(\frac{(\gamma+\mu)}{\beta}-3\right)}{2 \frac{(\gamma+\mu)}{\mu}\left(1-\frac{\alpha \gamma}{(\alpha+\mu)(\gamma+\mu)} \phi\right)}\right]^{2}+\left[\frac{N^{2} \mu\left(\frac{(\gamma+\mu)}{\beta}-1\right)}{2 \frac{(\gamma+\mu)}{\mu}\left(1-\frac{\alpha \gamma}{(\alpha+\mu)(\gamma+\mu)}\right)}\right]},
\end{aligned}
$$


and the solution of the extinction of one of the strains is as follows

$$
\begin{aligned}
& I_{1}^{*}=\frac{\mu N(\beta-(\gamma+\mu))}{(\gamma+\mu) \beta} \\
& I_{2}^{*}=0 .
\end{aligned}
$$

Finally, the stationary value of $R^{*}$, when hosts have been recovered from both strains, is given by the balance equation for the total population size $N$, explicitly

$$
R^{*}=N-\left(S^{*}+I_{1}^{*}+I_{2}^{*}+R_{1}^{*}+R_{2}^{*}+S_{1}^{*}+S_{2}^{*}+I_{12}^{*}+I_{21}^{*}\right) .
$$

These analytic results agree well with the numerical results from the time series analysis for small $\phi$ values, where the fixed point solution is stable. We will now continue with the bifurcation analysis beyond this fixed point solution using the continuation method.

\subsection{Bifurcations analysis beyond equilibria}

We investigate in detail the region of interest of $\phi<1$ for $\alpha=2$. All other parameter values are fixed and given in Table 1. In Figure 10 a) the bifurcation diagram by continuation, obtained with the numerical software AUTO [10], is shown for the interval of $0 \leq \phi \leq 1.1$ for the logarithm of the total number of infected $I$. As opposed to the previous bifurcation diagrams (Figs. 5 and 6), where all local extrema where shown, AUTO only gives for the limit cycles the global extrema.

In Fig. 10 a) we see that the fixed equilibrium becomes unstable at a supercritical Hopf bifurcation $H$ where a stable fixed limit cycle originates. The Hopf bifurcation appears at $\phi=0.1133$. This stable limit cycle becomes unstable at a pitchfork bifurcation point $P^{-}$for a limit cycle at $\phi=0.4114$. Solid lines denote stable equilibria or limit cycles, dashed lines unstable equilibria or periodic-one limit cycles. Thin lines are the secondary limit cycles: long-dashed stable and dotted unstable.

This point marks the origin of a pair of S-conjugate stable limit cycles besides the now unstable fixed limit cycle in the following sense: The Eq. system (2.2) in the symmetric case, hence for $\beta_{1}=\beta_{2}=\beta$ and $\phi_{1}=\phi_{2}=\phi$ is $\mathbb{Z}^{2}$-symmetric [21]. With a symmetry transformation matrix $\mathbf{S}$

$$
S:=\left(\begin{array}{cccccccccc}
1 & 0 & 0 & 0 & 0 & 0 & 0 & 0 & 0 & 0 \\
0 & 0 & 1 & 0 & 0 & 0 & 0 & 0 & 0 & 0 \\
0 & 1 & 0 & 0 & 0 & 0 & 0 & 0 & 0 & 0 \\
0 & 0 & 0 & 0 & 1 & 0 & 0 & 0 & 0 & 0 \\
0 & 0 & 0 & 1 & 0 & 0 & 0 & 0 & 0 & 0 \\
0 & 0 & 0 & 0 & 0 & 0 & 1 & 0 & 0 & 0 \\
0 & 0 & 0 & 0 & 0 & 1 & 0 & 0 & 0 & 0 \\
0 & 0 & 0 & 0 & 0 & 0 & 0 & 0 & 1 & 0 \\
0 & 0 & 0 & 0 & 0 & 0 & 0 & 1 & 0 & 0 \\
0 & 0 & 0 & 0 & 0 & 0 & 0 & 0 & 0 & 1
\end{array}\right)
$$




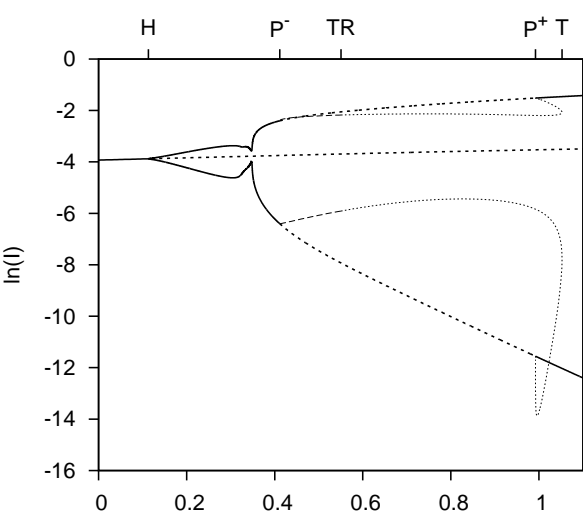

a)

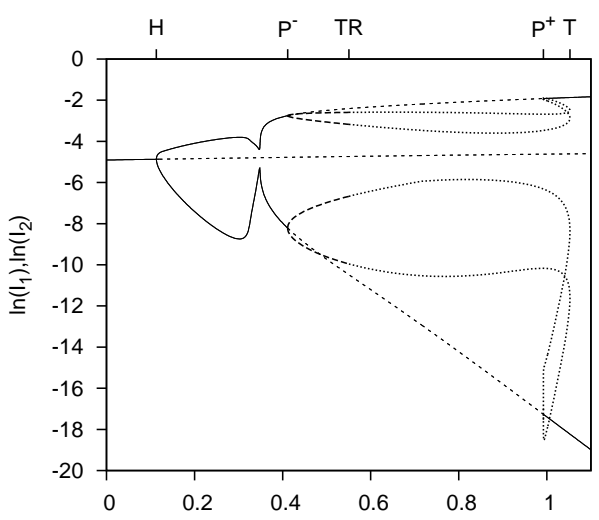

b)

Figure 10: a) Equilibria or maximum and minimum values for limit cycles of the logarithm for the total number of infected. We find a Hopf bifurcation at $H$ at $\phi=0.1133$, pitchfork (multiplier 1) bifurcations $P^{-}$at $\phi=0.4114$ and $P^{+}$at $\phi=0.9921$, torus bifurcation $T R$ at $\phi=0.5507$ and tangent bifurcation $T$ at $\phi=1.0524$. b) Equilibria or maximum and minimum values for limit cycles for $I_{1}$ and $I_{2}$. On the primary branch we have $\tilde{I}_{1}(t)=\tilde{I}_{2}(t), \tilde{R}_{1}(t)=\tilde{R}_{2}(t), \tilde{S}_{1}(t)=\tilde{S}_{2}(t)$ and $\tilde{I}_{12}(t)=\tilde{I}_{21}(t)$, for times $t$ up to the period length of the limit cycle. On the secondary branch two stable limit cycles coexist because of the symmetry.

for an equilibrium point $\mathbf{S} \underline{x}^{*}=\underline{x}^{*}$ holds, the state being defined by Eq. (3.2). Then this equilibrium is called fixed (see [21]). For limit cycles a similar terminology holds. A periodic solution is called fixed (see [21]) when $\mathbf{S} \underline{\tilde{x}}(t)=\underline{\tilde{x}}(t)$ and the associated limit cycles are also called fixed. There is another type of periodic solution that is not fixed but called symmetric when

$$
\mathbf{S} \underline{\tilde{x}}(t)=\underline{\tilde{x}}\left(t+\frac{T}{2}\right)
$$

where $T$ is the period, hence the limit cycle is shifted by half a period length. Again the associated limit cycles are also called symmetric. Both types of limit cycles $L$ are $\mathbf{S}$-invariant as curves: $\mathbf{S} L=$ $L$. An $\mathbf{S}$-invariant cycle is either fixed or symmetric. Two noninvariant limit cycles $(\mathbf{S} L \neq L)$ are called S-conjugate if their corresponding periodic solutions satisfy $\underline{\tilde{y}}(t)=\mathbf{S} \underline{\tilde{x}}(t)$ for all times $t$.

Figure $10 \mathrm{~b}$ ) gives the results for the infected with a single strain $I_{1}$ and $I_{2}$. Because these two variables are interchangeable this can also be interpreted as the stable limit cycles for the single variable say $I_{1}$. The fixed stable equilibrium below the Hopf bifurcation where we have $I_{1}^{*}=I_{2}^{*}$, $R_{1}^{*}=R_{2}^{*}, S_{1}^{*}=S_{2}^{*}$ and $I_{12}^{*}=I_{21}^{*}$ is a fixed equilibrium. For the fixed limit cycle in the parameter interval between the Hopf bifurcation and the pitchfork bifurcation we have $\tilde{I}_{1}(t)=\tilde{I}_{2}(t), \tilde{R}_{1}(t)=$ $\tilde{R}_{2}(t), \tilde{S}_{1}(t)=\tilde{S}_{2}(t)$ and $\tilde{I}_{12}(t)=\tilde{I}_{21}(t)$. This means that at the Hopf bifurcation $H$ the stable fixed equilibrium point becomes an unstable fixed equilibrium point.

In the parameter interval between the two pitchfork bifurcations, two stable limit cycles coexist and these limit cycles are S-conjugate. At the pitchfork bifurcation points the fixed limit cycle becomes unstable and remains fixed, and two stable S-conjugate limit cycles originate (see [21, Theorem 7.7]). 
Table 2: List of bifurcations.

\begin{tabular}{|c|c|}
\hline Bifurcation & Description \\
\hline \multirow[t]{3}{*}{$H$} & Hopf bifurcation \\
\hline & equilibrium becomes unstable \\
\hline & origin of stable limit cycle \\
\hline \multirow[t]{4}{*}{$T$} & Tangent bifurcation \\
\hline & bifurcation of limit cycle \\
\hline & one multiplier $=1$ \\
\hline & collision of two limit cycles \\
\hline \multirow[t]{4}{*}{$P$} & Pitchfork bifurcation \\
\hline & bifurcation of limit cycle \\
\hline & one Floquet multiplier $=1$ \\
\hline & origin of two secondary stable limit cycle branches \\
\hline \multirow[t]{4}{*}{$F$} & Flip bifurcation or period doubling bifurcation \\
\hline & bifurcation of limit cycle \\
\hline & one Floquet multiplier $=-1$ \\
\hline & origin of a limit cycle with double period length \\
\hline \multirow[t]{3}{*}{$T R$} & Torus bifurcation \\
\hline & bifurcation of limit cycle \\
\hline & $\begin{array}{l}\text { pair of complex conjugate multipliers with magnitude } 1 \\
\text { origin of an invariant torus }\end{array}$ \\
\hline
\end{tabular}

The invariant plane $I_{1}=I_{2}, R_{1}=R_{2}, S_{1}=S_{2}, I_{12}=I_{21}$ forms the separatrix between the pair of stable S-conjugate limit cycles $\underline{\tilde{x}}(t)$ and $\mathbf{S} \underline{\tilde{x}}(t)$ for all times $t$. The initial values of the two state variables $S(0)$ and $R(0)$ together with the point on the invariant plane, determine to which limit cycle the system converges.

Continuation of the stable symmetric limit cycle gives a torus bifurcation or Neimark-Sacker bifurcation at the parameter point denoted by $T R$ at $\phi=0.5507$. At his point the limit cycles become unstable because a pair of complex-conjugate Floquet multipliers crosses the unit circle. Floquet multipliers replace in the stability analysis of limit cycles (Floquet theory) often the eigenvalues used to analyse fixed point stability [21]. In [4] a sequence of Neimark-Sacker bifurcations into chaos is mentioned as one possible route to chaos.

Increasing the bifurcation parameter $\phi$ along the now unstable pair of S-conjugate limit cycles leads to a tangent bifurcation $T$ where a pair of two unstable limit cycles collide. This branch terminates at the second pitchfork bifurcation point denoted by $P^{+}$at $\phi=0.9921$. Because the first fold point gave rise to a stable limit cycle and this fold point to an unstable limit cycle we call the first pitchfork bifurcation supercritical and the latter pitchfork bifurcation subcritical.

These results agree very well with the simulation results shown in the bifurcation diagram for the maxima and minima of the overall infected in Figure 11. Notice that AUTO calculates only the global extrema during a cycle, not the local extrema. 


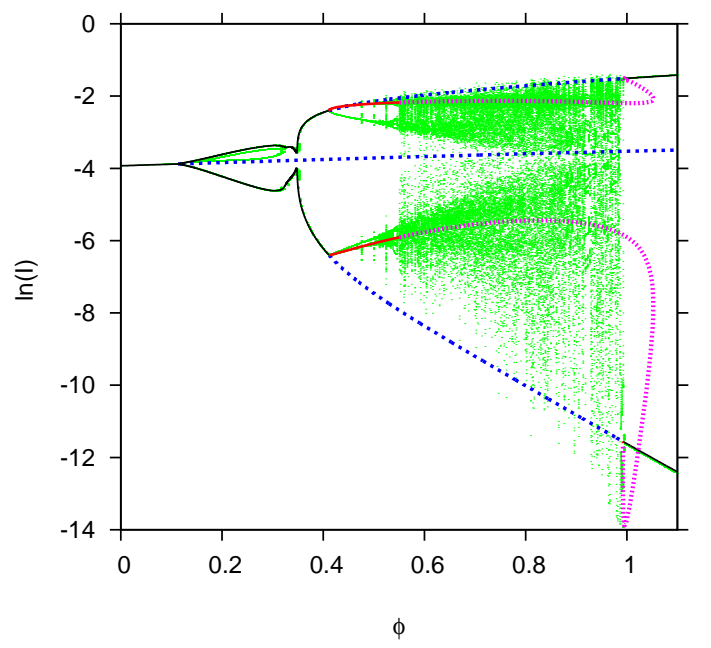

Figure 11: Comparison between bifurcation analysis by continuation from Fig. 10 a) (colored lines) and the numerical bifurcation diagram (green dots) as part for $\phi<1.1$ from Fig. 5. The overall bifurcation structure agrees well between both methods.

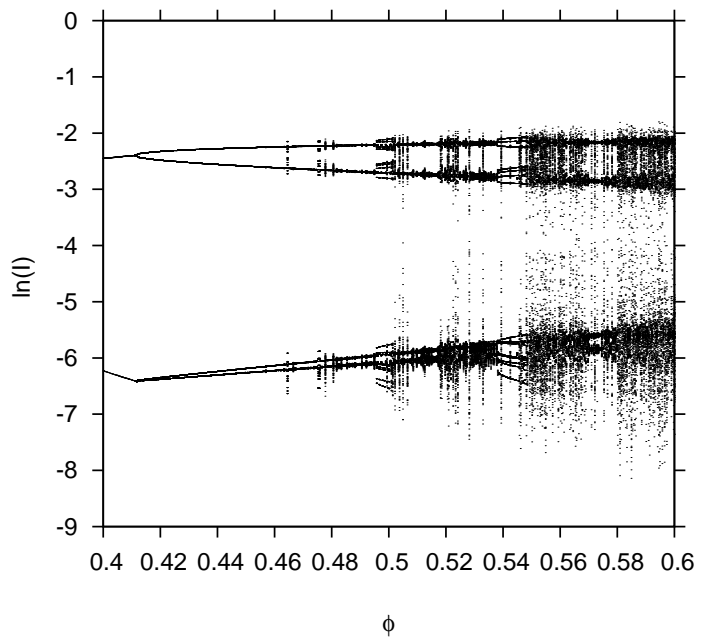

Figure 12: Detailed bifurcation diagram with high resolution in integration, transients and bifurcation parameter $\phi$, here for $\alpha=2$ and for $\phi$ between 0.4 and 0.6. Besides chaotic attractors respectively long chaotic transients also complicated limit cycles appear already for $\phi$ values around 0.50 , long before the torus bifurcation for $\phi$ around 0.55 . 
The previous results have been obtained by continuation starting from the fixed point solutions, Eq. system (4.1), tracking after the first Hopf bifurcation the limit cycles and their bifurcations, until new dynamical structures like toruses appear. The main bifurcation structures can be understood in comparison between the numerical bifurcation diagram and the present results in Figure 11. However, with the time series analysis shown in the previous section, more is observed, especially positive Lyapunov exponents appear around or even before the torus bifurcation.

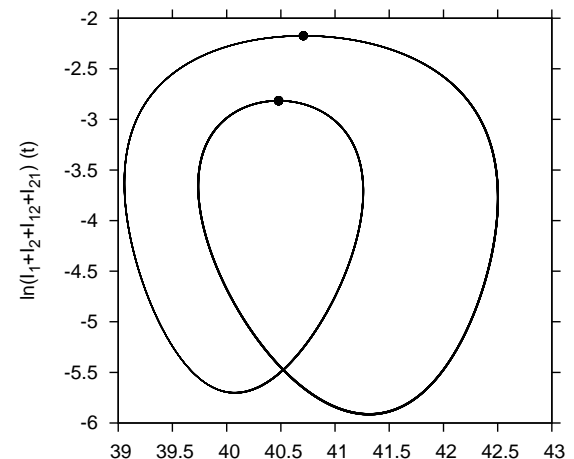

a)

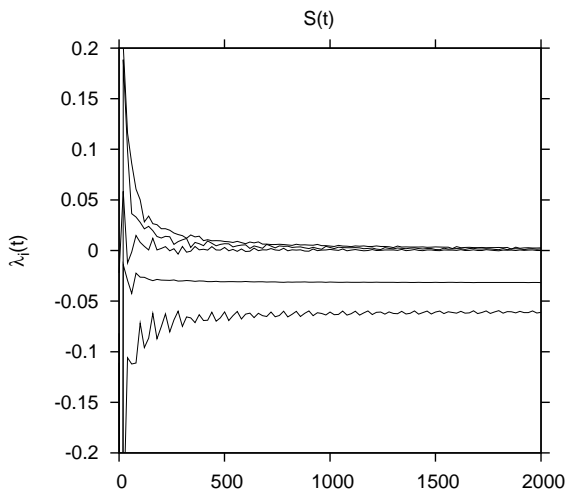

c)

Figure 13: a) State space plot for $\phi=0.5504$, in the region of previously unexplained co-existences of limit cycles, torus bifurcations and attractors with positive Lyapunov exponents. Special initial conditions were taken, to obtain the simple limit cycle found in numeric bifurcation analysis. b) Same parameter values, but arbitrary initial conditions. The attractor looks chaotic. c) Calculation of Lyapunov exponent along the limit cycle shown in a). The largest Lyapunov goes to zero, as do the next two due to closeness to a bifurcation. d) Same as in c), but with arbitrary initial conditions. The largest Lyapunov exponent converges to a value significantly larger than zero, the second towards zero. Hence the attractor in b) is chaotic.

We investigate in detail a region for $\alpha=2$ and $\phi$ around 0.55 where AUTO found limit cycles and torus bifurcations via continuation methods from earlier detected limit cycles, but where also more complicated attractors appear as a more detailed bifurcation diagram with arbitrary initial conditions reveals in Fig. 12.

We then search for the respective state space structures, see Fig. 13. In Fig. 13 a) the state space plot for $\phi=0.5504$ shows a limit cycle, as predicted by the continuation method. This 
is the region where the bifurcation diagram by continuation initially gives different results, limit cycles and torus bifurcation, from the bifurcation analysis by time series methods and the analysis of Lyapunov exponents, where already a positive Lyapunov exponent appears. For the limit cycle in Fig. 13 a) and c) special initial conditions were taken, as obtained from the analysis with AUTO. For this limit cycle given in Fig. 13 a) we find a zero largest Lyapunov exponent, see Fig. 13 c). The next two Lyapunov exponents also around zero indicate that we are close to a bifurcation point of this limit cycle, the soon coming torus bifurcation.

However, when taking arbitrary initial conditions we find for the same parameter values of the model as used in Fig. 13 a), especially the same $\phi$ value, the attractor shown in $13 \mathrm{~b}$ ). This attractor shows a largest Lyapunov exponent significantly larger than zero, see Fig. $13 \mathrm{~d}$ ). The second largest Lyapunov exponent converges to zero, as expected for a non-equilibrium attractor.

For values of $\phi$ slightly smaller than 0.5504 , the same analysis shows co-existing limit cycles (from which the attractor in Fig. 13 b) originates). Tracing such a limit cycle by AUTO finally gives new isola solutions in the analysis performed by AUTO, which previously have been missed by continuation starting at the equilibria and via the first Hopf-bifurcation. An isola is an isolated solution branch of limit cycles [15]. These isola cycles $L$ are not $\mathbf{S}$-invariant, that is $\mathbf{S} L \neq L$. The new isolas are shown relatively to the previously obtained bifurcation diagram by continuation in Fig. 14 a).

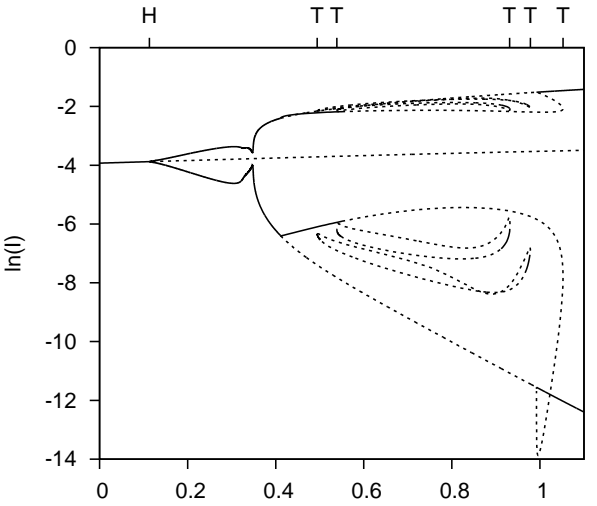

a)

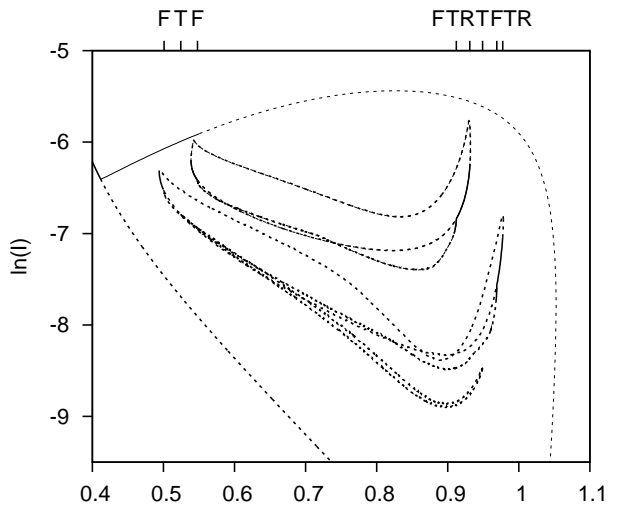

b)

$\phi$

Figure 14: a) Equilibria or maximum and minimum values for limit cycles for total infected, now including the new isolas between tangent bifurcations $T$ at $\phi=0.4941,0.5387,0.9310,0.9783$ and 1.0524. These new isolas are found starting at $\phi$ values smaller than the torus bifurcation. b) Isola bifurcations in more detail: tangent bifurcations $T$ at $\phi=0.5245$ and 0.9491 , torus bifurcations $T R$ at $\phi=0.9310$ and 0.9773 and flip bifurcations $F$ at $\phi=0.5009,0.5479,0.9120$ and 0.9691 . Some of the tangent bifurcations are not indicated in the plot (namely $T$ at $\phi=0.4941,0.5387$, $0.9310,0.9783,1.0524)$.

In Fig. 14 b) we investigate in more detail these isolas, obtaining flip or period doubling bifurcations and further torus bifurcations. These period doubling bifurcation sequences indicate another route to chaos than the previously found torus bifurcation. These results suggest that for these isolas two classical routes to chaos exist, namely via the torus or Neimark-Sacker bifurcation 
where the dynamics on the originating torus is chaotic, and the cascade of period doubling route to chaos. Two windows with period solutions within the chaotic windows, see Fig. 11, are filled by the two stable limit cycles of the isolas shown in Fig. 14. The study of the two windows with zero largest Lyapunov exponent shown in Fig. 9 is beyond the scope of this paper.

In order to obtain further insight into the possible bifurcation structures for the model under investigation we also looked at other parameter values in the symmetric and also briefly the asymmetric case. For other values of $\alpha$ in the region between 1 and 3 we found a period doubling route to chaos as well as the torus bifurcation already mentioned for $\alpha=2$.

The bifurcation analysis presented here was only possible in close comparison between the different tools presented here: a) the bifurcation analysis by continuation, giving accurate bifurcation points and classifications due to the analysis of the stability changes via Floquet multipliers, b) direct numerical bifurcation plots, revealing co-existing dynamic structures which continuation easily misses, and c) Lyapunov exponent calculations. The analysis even for the symmetric case with $\alpha=2$ is not exhaustive, more co-existing structures might appear when zooming further into the parameter space. But we obtained a good agreement between the different methods for the overall sketch of the dynamic complexity in the region of interest of $\phi<1$ in the symmetric case.

We also looked at numerical bifurcation diagrams for some asymmetric cases $\phi_{1} \neq \phi_{2}$ and $\beta_{1} \neq \beta_{2}$, which already indicated similarly if not more complicated bifurcation structures (not shown here). Future work on the relevant parameters for dengue epidemiology will be needed to identify eventual deviations from the simplest symmetric case investigated here.

\section{Conclusion}

Our analysis showed deterministically chaotic attractors for a multi-strain model in an unexpected parameter region just by adding temporary cross-immunity to previously existing dengue models.

Our model is a basic two-strain SIR-type model for the host population and was motivated by modelling dengue fever epidemiology with its peculiar ADE phenomenology. The simple structure of the model allows to generalize our findings to other multi-strain epidemiological systems, capturing the effective dynamics of the human host population. We could find deterministic chaos in a very basic model with only two strains and one reinfection possible, not needing the strong ADE mechanism, but rather stating that upon second infection hosts spread a disease less likely, since it might be more harmful and hence lead to hospitalization.

In this work, we focused on the multi-strain aspect and its effects on the host population, taking effects of the vector dynamics or seasonality only into account by the effective parameters in the SIR-type model, but not modelling these mechanisms explicitly. Since seasonally forced SIR systems can show already deterministic chaos [31], we expect that rather more complex dynamics will appear.

For such scenarios new tools of non-linear data analysis like Takens' embedding are available $[27,32]$, and allow to obtain topological information (fixed points, periodic orbits and the nature of chaotic attractors) about the whole multi-strain epidemiological system from time series of overall infecteds only, not needing any single strain data sets. 
This indicates that deterministic chaos is much more important in multi-strain models than previously thought, and opens new ways to data analysis of existing dengue time series.

\section{Acknowledgements}

We would like to thank Gabriela Gomes, Lisbon, for valuable discussions on multi-strain dynamics, Francisco Lemos and Sônia Diniz, Belo Horizonte, Minas Gerais, and Scott Halstead, Bethesda, Maryland, for detailed information about dengue epidemiology.

\section{References}

[1] B. Adams, M. Boots. Modelling the relationship between antibody-dependent enhancement and immunological distance with application to dengue. Jounal of Theoretical Biology, 242 (2006), 337-46.

[2] B. Adams, M. Boots. The influence of immune cross-reaction on phase structure in resonant solutions of a multi-strain seasonal SIR model. Jounal of Theoretical Biology, 248 (2007), 202-11.

[3] M. Aguiar,N. Stollenwerk. A new chaotic attractor in a basic multi-strain epidemiological model with temporary cross-immunity. (2007) arXiv:0704.3174v1 [nlin.CD].

[4] D. Albers, J. Sprott. Routes to chaos in high-dimensional dynamical systems: A qualitative numerical study. Physica D, 223 (2006), 194-207.

[5] D. Alonso, A. McKane, M. Pascual. Stochastic Amplification in Epidemics. Journal of the Royal Society Interface, 4 (2006), 575-582.

[6] L. Billings, B.I. Schwartz, B.L. Shaw, M. McCrary, D.S. Burke, T.A.D. Cummings. Instabilities in multiserotype disease models with antibody-dependent enhancement. Journal of Theoretical Biology, 246 (2007), 18-27.

[7] L. Chow, S. T. Hsu. MAC-ELISA for the detection of IgM antibodies to dengue type I virus. Journal of Clinical Microbiology, 22 (1989), 278-85.

[8] W.V. David. Invited Commentary: Dengue Lessons from Cuba. Am. J. Epidemiol., 152 (2000), 800-03.

[9] W.V. David, S. Green, S. Kalayanarooj, L.B. Innis, S. Nimmannitya, S. Suntayakorn, P.T. Endy, B. Raengsakulrach, L.A. Rothman, A.F. Ennis, A. Nisalak. Dengue viremia titer, Antibody Response Pattern, and Virus Serotype Correlate with Disease Severity. J. Infect. Dis., 181 (2000), 2-9.

[10] E.J. Doedel, R.C. Paffenroth, A.R. Champneys, T.F. Fairgrieve, Y.A. Kusnetsov, B. Sandstede, B. Oldeman, X.J. Wang, C. Zhang, AUTO 07P - Continuation and bifurcation software for ordinary differential equations, Technical Report: (2007) Concordia University, Montreal, Canada, and http://indy.cs.concordia.ca/auto/. 
[11] J.P. Eckmann, S. Oliffson-Kamphorst, D. Ruelle, S. Ciliberto. Liapunov exponents from time series. Phys. Rev A, 34 (1986), 4971-9.

[12] J.D. Farmer, J.J. Sidorowich. Predicting chaotic time series. Phys. Rev. Lett., 95 (1987), 845848.

[13] N. Ferguson, R. Anderson, S. Grupta. The effect of antibody-dependent enhancement on the transmission dynamics and persistence of multiple-strain pathogens. Proc. Natl. Acad. Sci. USA, 96 (1999), 790-94.

[14] D.B. Fischer, S.B. Halstead. Observations related to pathogenesis of dengue hemorrhagic fever. $V$. Examination of age specific sequential infection rates using a mathematical model. J. Biol. Med., 42 (1970), 329-49.

[15] M. Golubitsky, D.G. Schaeffer. Singularities and groups in bifurcation theory. Springer, New York, 1985.

[16] M.G. Guzmán, G. Kourí, L. Valdés, J. Bravo, M. Alvarez, S. Vazques, I. Delgado, B.S. Halstead. Epidemiologic Studies on Dengue in Santiago de Cuba, 1997. Am. J. Epidemiol., 152 (2000), 793-99.

[17] S.B. Halstead, E.J. Rourke. Dengue viruses and mononuclear phagocytes I: infection enhancement by non neutralizing antibody. J. Exp. Med., 146 (1977), 201-17.

[18] S.B. Halstead. Immune enhancement of viral infection. Progress in Allergy. Progress in Allergy, 31 (1982), 301-64.

[19] S.B. Halstead. Neutralization and antibody-dependent enhancement of dengue viruses. Advances in Virus Research, 60 (2003), 421-67.

[20] S.C. Kliks, A. Nisalak, W.E. Brandt, L. Wahl, D.S. Burke. Antibody-dependent enhancement of dengue virus growth in human monocytes as a risk factor for dengue hemorragic fever. Am. J. Trop. Med. Hyg., 40 (1989), 444-51.

[21] Y.A. Kuznetsov. Elements of Applied Bifurcation Theory. No. 112, Applied Mathematical Sciences. Springer-Verlag, 3 edition, New York, 2004.

[22] Pers comm.: Francisco Lemos, Zoonosis Control Coordinator of Secretaria de Estado de Saúde de Minas Gerais, Brazil; Sônia Diniz, Responsible of the virology and rickettsioses service from the Fundao Ezequiel Dias, Minas Gerais, Brazil, \& Scott Halstead, Director of Pedriatic Dengue Vaccine Initiative, Bethesda, Maryland, USA.

[23] Y.W. Lin, K.J. Wang, H.Y. Lei, M.T. Lin, S.H. Liu, C.C. Liu, H.S. Chen. Virus Replication and Cytokine Production in Dengue Virus-Infected Human B Lymphocytes. Journal of Virology, 76 (2002), 1242-49.

[24] S. Matheus, X. Deparis, B. Labeau, J. Lelarge, J. Morvan, P. Dussart. Discrimination between Primary and Secondary Dengue Virus Infection by an Immunoglobulin G Avidity Test Using a Single Acute-Phase Serum Sample. Journal of Clinical Microbiology, 43 (2005), 2793-97.

[25] A.J. McKane, T.J. Newman. Predator-prey cycls from resonant amplification of demographic stochasticity. Phys. Rev. Lett., 94 (2005), 218102-7. 
[26] E. Ott. Chaos in Dynamical Systems. Cambridge University Press, Cambridge, 2002.

[27] N.H. Packard, J.P. Crutchfield, J.D. Farmer, R.S. Shaw. Geometry from a Time Series. Phys. Rev. Lett., 45 (1980), 712-716.

[28] U. Parlitz. Identification of true and spurious Lyapunov exponents from time series. Int. J. Bif. Chaos., 2 (1992), 155-165.

[29] D. Ruelle. Chaotic Evolution and Strange Attractors. Cambridge University Press, Cambridge, (1989).

[30] I.B. Schwartz, L.B. Shaw, D.A.T. Cummings, L. Billings, M. McCrary, D.S. Burke. Chaotic desynchronization of multi-strain diseases. Physical Review E, 72 (2005), 066201-6.

[31] L. Stone, R. Olinky, A. Huppert. Seasonal dynamics of recurrent epidemics. Nature, 446 (2007), 533-36.

[32] F. Takens. Detecting strange attractors in turbulence. in: Dynamical Systems and Turbulence, Warwick 1980 eds. D. Rand, L.S. Young. Lecture Notes in Mathematics. (1981), No. 898, Springer, Berlin.

[33] H.J. Wearing, P. Rohani. Ecological and immunological determinants of dengue epidemics. Proc. Natl. Acad. Sci. USA, 103 (2006), 11802-11807.

[34] R.M. Welsh, L.K. Selin. No one is naive: The significance of heterologous T-cell immunity. Nature Reviews in Immunology, 2 (2002), 417-426.

[35] World Health Organization. Dengue and Dengue Hemorrhagic Fever. (World Health Org., Geneva (2002), Fact Sheet 117). 